\begin{tabular}{|c|c|}
\hline Title & $\begin{array}{l}\text { Superior Microvascular Perfusion of Infused Liposome Encapsulated Hemoglobin Prior to Reductions in Infarctions } \\
\text { after Transient Focal Cerebral Ischemia }\end{array}$ \\
\hline Author(s) & $\begin{array}{l}\text { Shimbo, Daisuke; A bumiya, Takeo; Kurisu, Kota; O sanai, Toshiya; Shichinohe, Hideo; Nakay ama, Naoki; Kazumata, } \\
\text { Ken; Nakamura, Hideki; Shimuzu, Hiroshi; Houkin, Kiyohiro }\end{array}$ \\
\hline Citation & $\begin{array}{l}\text { Journal of stroke \& cerebrovascular diseases, 26(12), } 2994.3003 \\
\text { https://doi.org/10.1016/.jstrokecerebrovasdis.2017.07.026 }\end{array}$ \\
\hline Issue Date & 2017-12 \\
\hline Doc URL & http:/hdl .handle.net/2115//2077 \\
\hline Rights & $\begin{array}{l}\text { () 2017. This manuscript version is made available under the CC-BY-NC-ND } 4.0 \text { license } \\
\text { http://reativecommons.org/icenses/oy-nc-nd/4.0/ }\end{array}$ \\
\hline Rights(URL) & http://creativecommons.org/icenses/by-nc-nd/4.0/ \\
\hline Type & article (author version) \\
\hline File Information & JStrokeCerebrovascDis26_2994.pdf \\
\hline
\end{tabular}

Instructions for use 


\section{Superior microvascular perfusion of infused liposome-encapsulated hemoglobin prior to reductions in infarctions after transient focal cerebral ischemia}

\section{Abstract}

Background: The Development of cerebral infarction after transient ischemia is attributed to post-ischemic delayed hypoperfusion in the microvascular region. In the present study, we assessed microvascular perfusion capacity of infused liposome-encapsulated hemoglobin (LEH) in a therapeutic approach for transient middle cerebral artery occlusion (tMCAO). Methods: Two-hour MCAO rats were immediately subjected to intra-arterial infusion of LEH (LEH group) or saline (vehicle group) or no treatment (control group), and then to recanalization. Neurological findings, infarct and edema progression, microvascular endothelial dysfunction, and inflammatory reactions were compared between the three groups after 24 hours of reperfusion. Microvascular perfusion in the early phase of reperfusion was evaluated by hemoglobin immunohistochemistry and transmission electron microscopy. Results: The LEH group achieved significantly better results in all items evaluated than the other groups. Hemoglobin immunohistochemistry revealed that the number of hemoglobin-positive microvessels was significantly greater in the LEH group than in 
the other groups $(\mathrm{P}<0.01)$, with microvascular perfusion being more likely in narrow microvessels $(\leq 5 \mu \mathrm{m}$ in diameter). An electron microscopic examination revealed that microvessels in the control group were compressed and narrowed by swollen astrocyte end-feet, whereas those in the LEH group had a less deformed appearance and contained LEH particles and erythrocytes. Conclusion: The results of the present study demonstrated that the infusion of LEH reduced infarctions after tMCAO with more hemoglobin-positive and less deformed microvessels at the early phase of reperfusion, suggesting that the superiority of the microvascular perfusion of LEH mediates its neuroprotective effects.

Key words: infarction, transient focal cerebral ischemia, microvessels, neuroprotection 
Stroke is a major cause of death and disability in many developed nations. The recent establishment of revascularization therapies for acute ischemic stroke may result in more favorable outcomes than those previously achieved. However, ischemia-reperfusion (I/R) after revascularization therapies sometimes causes tissue damage that develops after vascular recanalization due to the pre-existence of a prolonged and severe ischemic period. ${ }^{1}$ Based on animal experimental models, the development of infarction after transient ischemia is assumed to be due to post-ischemic delayed hypoperfusion in the microvascular region. Decreased capillary flow with capillary diameter reductions was demonstrated in delayed post-ischemic hypoperfusion using a video microscopic analysis. ${ }^{2}$ In addition, previous studies using electron microscopy reported that I/R induced astrocytic end-foot swelling, which transiently compressed and narrowed adjacent microvessels from a couple of hours to several hours after reperfusion. ${ }^{3,} 4$ Therefore, microvascular narrowing may contribute to post-ischemic delayed hypoperfusion.

Liposome-encapsulated hemoglobin (LEH) was originally developed as a blood substitute using technologies for the encapsulation of concentrated hemoglobin with liposomes in order to overcome issues associated with blood transfusion. ${ }^{5}$ The mean diameter of LEH ranges between 200 and $250 \mathrm{~nm}$ (1/40th the size of erythrocytes), and 
this is expected to be advantageous for perfusion into microvessels. Therefore, LEH has been examined as a therapeutic agent for ischemic lesions with perfusion disturbances. Previous studies demonstrated the therapeutic effects of LEH using animal brain ischemic models. ${ }^{6-12}$ Another advantage of LEH as artificial blood is that it does not contain inflammatory cells. Since neutrophils cause inflammatory reactions, which lead to tissue damage after transient ischemia, namely, $\mathrm{I} / \mathrm{R}$ injury, the suppression of neutrophil function may be employed as a therapeutic strategy for I/R injury. ${ }^{13}$ The selective arterial infusion of LEH transfers oxygen to affected regions with reductions in the influx of neutrophils. In our previous study, we selectively infused LEH into the recanalized artery of a rat transient middle cerebral artery occlusion (tMCAO) model in order to ameliorate tissue damage induced by neutrophil-mediated inflammatory reactions. The selective infusion of LEH for 2 hours reduced the influx of autologous blood cells and ameliorated tissue damage in the reperfused area. ${ }^{14}$ In the present study, we focused on the microvascular perfusion capacity of LEH in order to investigate the neuroprotective effects of LEH in a rat tMCAO model. LEH was administrated from the recanalized internal carotid artery for a short-term period in order to confirm whether infused LEH is superior to autologous erythrocytes in terms of microvascular perfusion and if LEH superior perfusion precedes reductions in infarctions after tMCAO. 


\section{Material and Methods}

Animals

All animal experiments were approved by the Animal Studies Ethical Committee at the Hokkaido University Graduate School of Medicine. All procedures used in this study were performed in accordance with the institutional guidelines for animal experiments..

\section{$L E H$}

LEH was developed, manufactured, and provided by Terumo Corporation (Tokyo, Japan). The relevant characteristics of LEH have been reported. ${ }^{5}$ LEH contains inositol hexaphosphate, which is used as an allosteric effector to modulate the oxygen affinity of $\mathrm{Hb}$ over that of usual erythrocytes. The average size of liposomes ranges between 200 and $250 \mathrm{~nm}$, which is $1 / 40$ th the size of erythrocytes. The liposomal surface is modified polyethylene glycol to increase LEH stability during storage and use in blood by preventing aggregation. LEH was suspended in saline at a hemoglobin concentration of $6 \mathrm{~g} / \mathrm{dl}$. LEH was oxygenated to a $\mathrm{PaO}_{2}$ of $110 \pm 10 \mathrm{mmHg}$ by mixing with room air prior to use. 
MCAO Model and Experimental Groups

Male Sprague-Dawley rats (CLEA Japan, Inc., Tokyo, Japan), weighing between 260 and 300 g, were anesthetized using $4.0 \%$ isoflurane in $\mathrm{N}_{2} \mathrm{O}: \mathrm{O}_{2}(70: 30)$ and maintained with $2.0 \%$ isoflurane in $\mathrm{N}_{2} \mathrm{O}: \mathrm{O}_{2}(70: 30)$ through a facial mask. Focal cerebral ischemia was induced by right MCAO using a modified method of intraluminal suture occlusion. $^{14,} 15$ Regional cerebral blood flow was measured using laser Doppler flowmetry (OMEGA FLOW FLO-C1; OMEGAWAVE, Tokyo, Japan) before and after MCAO in order to confirm successful MCAO. Rats that did not show a regional blood flow reduction to $30 \%$ of the pre-ischemic baseline were excluded from further experimentation. Rats subjected to 2-hour MCAO were divided into 3 groups, 1) an LEH group infused with LEH (20 ml/kg) through a catheter for 15 minutes via the ICA, 2) a vehicle group infused with saline in the same manner as the LEH group, and 3) a control group subjected to recanalization only without the infusion. Infused LEH and saline were warmed to $37^{\circ} \mathrm{C}$ before infusion to avoid hypothermic neuroprotective effects due to low-temperature fluid infusion. Rats were sacrificed 24 hours after reperfusion and brains were collected to examine pathological changes. 
A neurological evaluation was performed under awake conditions after MCAO or 24-hour reperfusion. Rats were rated on spontaneous movement, the symmetry of limb movements, forepaw outstretching, climbing, body proprioception, and responses to vibrissae touch for a score of 3 to 18 points. ${ }^{16}$

\section{Infarct and Edema Volume Measurement}

Cerebral infarction and edema were evaluated using the 2,3,5-triphenyltetrazolium chloride (TTC) staining of coronal brain sections (thickness of $2 \mathrm{~mm}$ ), as described previously. ${ }^{14}$ Infarct and brain edema volumes were quantified with a standard computer-assisted image analysis system (Image J 1.37v; NIH Bethesda, MD, USA). Infarct volume was calculated as a percent volume of the normal left hemisphere according to the following formula: (left hemisphere volume - right non-infarct volume)/left hemisphere volume $\times 100 \%$. Brain edema volume was calculated as a percent volume of the normal left hemisphere according to the following formula: (right hemisphere volume - left hemisphere volume)/left hemisphere volume $\times 100 \%$. 
The ipsilateral hemisphere was collected 24 hours after reperfusion and processed for a Western blot analysis as described previously. ${ }^{14}$ The first antibodies were an anti-myeloperoxidase (MPO) antibody (Cell Signaling Technology, Danvers, MA, USA, dilution 1:2000) as a marker for neutrophils, an anti-matrix metalloproteinase (MMP)-9 antibody (Cell Signaling Technology, Danvers, MA, USA, dilution 1:2000), and anti-intercellular molecule-1 (ICAM-1) antibody (Abcam, Cambridge, MA, USA, dilution 1:2000). Western blot bands were quantified by densitometry using an image analysis system (ImageJ 1.37v; NIH Bethesda, MD, USA)

\section{Gelatin Zymography}

Gelatin zymography to detect MMP-9 activity was performed as described previously. ${ }^{14}$ Areas of MMP-9 activity appeared as clear bands against a dark background, and gels were scanned and quantified by densitometry using an image analysis system (Image J 1.37v, NIH Bethesda, MD, USA).

\section{Evaluation of Evans Blue Extravasation}

The Evans blue extravasation assay was used to analyze blood-brain barrier (BBB) disruption. Immediately after reperfusion, $4 \mathrm{~mL} / \mathrm{kg}$ of $2 \%$ Evans blue (Wako, Osaka, 
Japan) in saline was administered intravenously. Twenty-four hours after reperfusion, rats were perfused with saline through the left ventricle to wash out intravascular Evans blue. The ipsilateral cerebral hemisphere was homogenized in $3 \mathrm{ml}$ of $50 \%$ trichloroacetic acid solution and then centrifuged (10000 rpm for 20 minutes). The supernatant was measured at $620 \mathrm{~nm}$ for absorbance using a spectrophotometer (TECAN Japan, Kanagawa, Japan). The tissue contents of Evans blue were quantified from a linear standard curve and expressed as micrograms per gram of brain tissue. ${ }^{17}$

\section{Immunohistochemistry}

Paraffin sections of the brain fixed in $4 \%$ paraformaldehyde were used for immunohistochemistry. Four-micrometer-thick coronal sections at the level of the striatum were prepared. The first antibodies used in the present study were an anti-MPO antibody (dilution 1:100 at $4^{\circ} \mathrm{C}$ overnight), anti-human hemoglobin monoclonal antibody (1:800 at room temperature for 1 hour, Institute of Immunology, Tokyo, Japan), and anti-rat hemoglobin polyclonal antibody (1:800 at room temperature for 1 hour, Mitsubishi Chemical Medicine, Tokyo, Japan). After the first antibody incubation under the conditions described above, sections were treated with the Envision polymer of the Dako EnVision+Kit (DakoCytomation, Glostrup, Denmark) for 1 hour. The DAB 
chromogen of the DAB Substitute Kit (DakoCytomation) was applied for 2 to 3 minutes in order to obtain a chromogenic signal.

\section{Analysis of Microvascular Perfusion of LEH}

In order to evaluate the microvascular perfusion of LEH in the I/R region at the early phase of reperfusion, immunohistochemistry with anti-human hemoglobin was performed on paraffin sections of the brain harvested after ending intra-arterial LEH infusion in the LEH group. Immunohistochemistry with anti-rat hemoglobin was also performed on paraffin sections of the brain harvested in the same manner to the control and vehicle groups in order to compare the microvascular perfusion of autologous erythrocytes with that of LEH. A quantitative analysis of hemoglobin-positive microvessels in hemoglobin immunohistochemistry was performed using ImageJ software (Image J 1.37v; NIH Bethesda, MD, USA). The number of hemoglobin-positive microvessels and size of microvessels were quantified with binary images processed by ImageJ software at 3 randomly selected $200 \times$ magnified fields in the ipsilateral cortex in the MCA territory area at the level of the striatum. The average number of hemoglobin-positive microvessels was used for the quantitative analysis. 


\section{Electron Microscopic Analysis}

Brains were collected after 3 hours of reperfusion and fixed in 2.5\% glutaraldehyde in $0.1 \mathrm{~mol} / \mathrm{L}$ cacodylate buffer to prepare specimens for transmission electron microscopy. Specimens collected from the peri-infarct area at the level of the striatum were washed in cacodylate buffer, post-fixed with $1 \%$ osmic acid, and stained en bloc with $0.5 \%$ uranyl acetate in 50\% ethanol. After dehydration through a graded ethanol series, the fragments were embedded in Epon812 and polymerized in an oven at $60^{\circ} \mathrm{C}$ for 3 days. Ultrathin sections (thickness of $60 \mathrm{~nm}$ ) were stained with 50\% alcoholic uranyl acetate and lead citrate. The ultrastructures of brain microvessels and their surrounding components were examined using a transmission electron microscope (JEM-1400; Jeol, Tokyo, Japan).

\section{Statistical Analysis}

All quantitative measurements were made by blinded investigators. Data were expressed as the mean \pm SD. Two-group comparisons were performed using the 2-tailed Student's t-test. Multiple comparisons were analyzed using a one-way ANOVA followed by the Tukey-Kramer test. Values of $P<.05$ were considered significant. 


\section{Results}

\section{Physiological Parameters}

Physiological parameters were monitored twice: at the beginning of MCAO and at the end of reperfusion. No significant differences were observed in physiological parameters between the three groups. Furthermore, no significant differences were observed in the percentage of cerebral blood flow reductions between the three groups after MCAO (Table 1).

\section{Neurological Function and Infarct Volumes}

While 18-point neurological scores at the beginning of MCAO were not significantly different between the three groups (Fig 1A), these scores were significantly better in the LEH group (14.2 \pm 0.97$)$ than in the control $(10.6 \pm 1.85)$ and vehicle groups $(11.5 \pm 1.41)$ after 24 hours of reperfusion $(P<.01$, Fig 1B). Infarct volumes were significantly smaller in the LEH group (25.4 $\pm 7.7 \%)$ than in the control $(42.4 \pm 8.7 \%)$ and vehicle (41.6 $\pm 5.2 \%)$ groups $(P<.01$, Fig $1 C)$. Edema volumes were significantly smaller in the 
LEH group (106.3 $\pm 2.9 \%)$ than in the control $(117.9 \pm 4.4 \%)$ and vehicle $(115.7 \pm 2.9 \%)$ groups $(P<.01$, Fig $1 \mathrm{C})$.

Neutrophil Recruitment

MPO immunohistochemistry revealed that MPO-positive neutrophils were recruited to the ischemic brain and extravasated into the perivascular space and parenchyma after 24 hours of reperfusion in the control and vehicle groups, but not in the LEH group (Fig 2A). MPO Western blotting showed that the expression of MPO was significantly weaker in the LEH group than in the control and vehicle groups $(P<.01$, Fig $2 \mathrm{~B})$.

\section{MMP-9 Expression and Activity}

The expression and activity of MMP-9 in the ischemic region after 24 hours of reperfusion were examined by Western blotting and zymography, respectively. MMP-9 Western blotting showed that its expression was increased in the control and vehicle groups, but not in the LEH group, resulting in a significant difference between these groups $(P<.01$, Fig 3A). Gelatin zymography showed that MMP-9 activity was also significantly lower in the LEH group than in the control group $(P<.01$, Fig 3B) and vehicle groups $(P<.05$, Fig 3B). 


\section{Endothelial Perturbation and Damage}

Endothelial perturbation and damage were evaluated by ICAM-1 expression and Evans blue extravasation. ICAM-1 Western blotting showed that its expression was increased in the control and vehicle groups, but not in the LEH group. The expression of ICAM-1 was significantly lower in the LEH group than in the control group $(P<.01$, Fig 4A) and vehicle groups $(P<.05$, Fig 4A). Evans blue extravasation was analyzed to assess BBB disruption. The content of Evans blue in the ischemic hemisphere was significantly lower in the LEH group than in the control group $(P<.01$, Fig 4B) and vehicle groups $(P<.05$, Fig 4B).

\section{Microvascular Perfusion of LEH}

The microvascular perfusion of $\mathrm{LEH}$ in the $\mathrm{I} / \mathrm{R}$ region was estimated in an immunostaining analysis with the anti-human hemoglobin antibody in the LEH group. Perfusion states were compared with those of autologous erythrocytes in immunostaining with the anti-rat hemoglobin antibody in the control and vehicle groups (Fig 5A). A quantitative analysis showed that the total number of hemoglobin-positive vessels was significantly greater in the LEH group than in the control and vehicle 
groups $(P<.01$, Fig 5B). The number of hemoglobin-positive narrow microvessels $(\leq 5$ $\mu \mathrm{m}$ in diameter) was significantly greater (4 to 8-fold) in the LEH group than in the control and vehicle groups $(P<.01$, Fig 5C).

\section{Microvascular Morphological Examination}

Microvascular morphological changes in the ischemic region were analyzed using brains harvested after 3 hours of reperfusion. We selected the time point of 3 hours of reperfusion because a previous electron microscopic analysis on $\mathrm{I} / \mathrm{R}$ injury demonstrated that microvascular morphological changes were enhanced at that time point. $^{3,4}$ Microvessels in the control group were frequently narrowed due to compression by swollen astrocyte end-feet, resulting in reductions in autologous erythrocytes in their lumen (Fig 6A). These microvascular morphological changes were less frequent in the LEH group, and, in addition, LEH particles and erythrocytes were observed together in the microvascular lumen (Fig 6B). A comparison of endothelial cells in the two groups revealed that the control group had a more swollen cytoplasm and more microvilli than the LEH group.

\section{Discussion}


In the present study, the intra-arterial infusion of LEH exerted neuroprotective effects with neurological improvements and infarct volume reductions. The LEH treatment suppressed inflammatory activation represented by neutrophil infiltration and MMP-9 production, and also suppressed endothelial perturbation and damage represented by ICAM-1 expression and Evans blue extravasation. The immunohistochemical analysis revealed the superiority of the microvascular perfusion of LEH, which was demonstrated by the number of hemoglobin-positive microvessels being significantly greater in the LEH group than in the other groups, with microvascular perfusion being more likely in narrow microvessels $(\leq 5 \mu \mathrm{m}$ in diameter). The electron microscopic analysis also revealed the superiority of the microvascular perfusion of LEH, which was demonstrated by microvessels in the LEH group showing less narrowed deformities than the control group and also simultaneously containing erythrocytes and LEH. These results suggest that LEH is perfused more effectively into microvessels and prevents capillary hypoperfusion, resulting in an effective oxygen supply to peripheral brain tissues.

We previously demonstrated that the post-ischemic intra-arterial infusion of LEH for 2 hours exerted neuroprotective effects against I/R injury in a rat tMCAO model. ${ }^{14}$ In that study, we selectively infused LEH into the recanalized artery in order to physically reduce 
the influx of neutrophils with the aim of mitigating I/R injury by inhibiting neutrophil inflammatory functions. However, we were unable to extend the LEH infusion time to more than 2 hours due to the difficulties associated with maintaining a steady anesthetic state in infused rats. Due to this experimental limitation, other neuroprotective mechanisms in addition to reductions in neutrophil inflow need to be considered in order to achieve LEH neuroprotective effects. Therefore, we focused on the microvascular perfusion capacity of LEH to investigate the neuroprotective effects of $\mathrm{LEH}$ in a rat tMCAO model in the present study.

We demonstrated that the superior microvascular perfusion of LEH was enhanced in narrow microvessels ( $\leq 5 \mu \mathrm{m}$ in diameter) in the immunohistochemical analysis. Since the mean diameter of rat erythrocytes is $6.5 \mu \mathrm{m},{ }^{18}$ it may be difficult for erythrocytes to pass through narrow microvessels $(\leq 5 \mu \mathrm{m}$ in diameter). The mean diameter of LEH ranges between 200 and $250 \mathrm{~nm}$ (1/40th the size of erythrocytes), which is advantageous for its perfusion into narrow microvessels. Although LEH was originally developed as a blood substitute, ${ }^{5}$ it has been examined as a therapeutic agent for ischemic stroke because of its structural characteristics as a small particle. Previous studies also showed the therapeutic effects of LEH using animal brain ischemic models, ${ }^{6-12}$ some of which confirmed the superior perfusion of LEH in the ischemic brain using imaging 
techniques. Urakami et al. reported that LEH was distributed from the periphery towards the core of ischemia in a rat MCAO model using positron emission tomography. ${ }^{12}$ Kawaguchi et al. demonstrated that LEH flowed more smoothly than autologous erythrocytes in small vessels using fluorescence microscopy. ${ }^{9}$ Taken together, the advantage of LEH in terms of microvascular perfusion appears to mediate its neuroprotective effects.

Another notable result of the present study was the ultrastructural morphological changes observed in microvessels in the I/R region in the electron microscopic analysis. In the control groups, microvessels become narrower due to the swelling of astrocyte end-feet and endothelial cells, resulting in the disappearance of erythrocytes in the rumen. Previous studies using electron microscopy demonstrated that I/R injury induced astrocytic end-foot swelling, which transiently compressed and narrowed adjacent microvessels from a couple of hours to several hours after reperfusion. ${ }^{3,4}$ Brain perfusion imaging analyses have not yet been performed on these microvascular morphological changes. However, one study using a carbon-black perfusion analysis demonstrated that microvascular perfusion evaluated by carbon-black filling transiently deteriorated in association with microvascular morphological changes from 3 to 8 hours after reperfusion. ${ }^{19}$ In addition to astrocytic end-foot swelling, endothelial swelling was 
demonstrated in the ischemic region during $\mathrm{MCAO},{ }^{20}$ as we observed in the control group in the present study. These endothelial changes also decreased the luminal area of microvessels. Based on these findings and our present results, we speculate that microvascular morphological changes contribute to post-ischemic delayed deterioration in the tMCAO model. ${ }^{21,22}$ The electron microscopic analysis in the LEH groups revealed that microvessels exhibited fewer narrowed deformities and simultaneously contained erythrocytes and LEH. These results under the LEH treatment also suggest the advantage of LEH for microvascular perfusion in the I/R region.

Microvascular sequential reactions are important in the formative process of I/R injury. Kurisu et al. demonstrated the following microvascular sequential reactions: an aquaporin-4 surge (2 hours), microvascular narrowing changes (2 to 6 hours), endothelial dysfunction (6 to 24 hours), and inflammatory activation (24 hours), in a time course analysis of the rat tMCAO model. ${ }^{4}$ Among these reactions, the LEH treatment inhibited endothelial dysfunction (the expression of ICAM-1 and Evans blue extravasation) and inflammatory activation (neutrophil infiltration and MMP-9 production) in the present study. Although neutrophil infiltration and MMP-9 production are significant processes in the development of $\mathrm{I} / \mathrm{R}$ injury, ${ }^{23,24}$ they are relatively late events that occur after 12 to 24 hours of reperfusion. ${ }^{4,25,26}$ Microvascular 
morphological changes and endothelial dysfunction were observed after 2 to 6 hours of reperfusion, ${ }^{4}$ which indicates that they occur upstream of inflammatory activation in the process of ischemia. Based on this temporal observation, the LEH treatment may initially ameliorate capillary hypoperfusion and subsequently suppress endothelial dysfunction. Since the half-life of LEH is approximately 10 hours, $^{5}$ the superior perfusion effects of LEH may be maintained during the transient development of microvascular narrowing changes from a couple of hours to several hours after reperfusion.

There are limitations in the present study. The neuroprotective effects of LEH against $\mathrm{I} / \mathrm{R}$ injury were only assessed in the acute phase. We need to examine the long-term outcomes of this LEH treatment. Furthermore, although the intra-arterial infusion of LEH was proven to be a useful strategy for preventing $I / R$ injury, we need to assess differences in the effects of LEH following its intra-arterial infusion and intra-venous infusion. The establishment of an appropriate dose is another issue. We need to perform a dose-escalation experiment in order to select a safe and effective dose range.

In conclusion, we herein demonstrated that the intra-arterial infusion of LEH exerted neuroprotective effects after transient ischemia, which resulted in the amelioration of capillary hypoperfusion and endothelial dysfunction. The superior microvascular 
perfusion of LEH was demonstrated in narrow microvessels in the immunohistochemical and electron microscopic analyses. Since this therapeutic strategy is convenient and feasible for clinical applications by an endovascular approach, it may be a promising candidate for preventing infarct enlargement with severe brain edema and hemorrhagic transformation after tissue plasminogen activator thrombolysis and/or endovascular thrombectomy.

\section{Conflict of interest}

The authors declare no competing financial interests

\section{Acknowledgments}

The authors thank Yumiko Shinohe for their technical assistance. 


\section{REFEREBCES}

1. Molina CA, Alvarez-Sabin J. Recanalization and reperfusion therapies for acute ischemic stroke. Cerebrovasc Dis 2009;27 Suppl 1:162-167

2. Hauck EF, Apostel S, Hoffmann JF, Heimann A, Kempski O. Capillary flow and diameter changes during reperfusion after global cerebral ischemia studied by intravital video microscopy. J Cereb Blood Flow Metab 2004;24:383-391

3. Ito U, Hakamata Y, Kawakami E, Oyanagi K. Temporary focal cerebral ischemia results in swollen astrocytic end-feet that compress microvessels and lead to delayed focal cortical infarction. J Cereb Blood Flow Metab 2011;31:328-338

4. Kurisu K, Abumiya T, Nakamura H, Shimbo D, Shichinohe H, Nakayama N, et al. Transarterial regional brain hypothermia inhibits acute aquaporin-4 surge and sequential microvascular events in ischemia/reperfusion injury. Neurosurgery 2016;79(1):125-134

5. Kaneda $\mathrm{S}$, Ishizuka $\mathrm{T}$, Goto $\mathrm{H}$, Kimura $\mathrm{T}$, Inaba $\mathrm{K}$, Kasukawa $\mathrm{H}$. Liposome-encapsulated hemoglobin, trm-645: Current status of the development and important issues for clinical application. Artif. Organs 2009;33:146-152

6. Hamadate N, Yamaguchi T, Sugawara A, Togashi H, Izumi T, Yoshida T, et al. Liposome-encapsulated hemoglobin ameliorates impairment of fear memory and hippocampal dysfunction after cerebral ischemia in rats. J Pharmacol Sci 2010;114:409-419

7. Kakehata J, Yamaguchi T, Togashi H, Sakuma I, Otani H, Morimoto Y, et al. Therapeutic potentials of an artificial oxygen-carrier, liposome-encapsulated hemoglobin, for ischemia/reperfusion-induced cerebral dysfunction in rats. J Pharmacol Sci 2010;114:189-197

8. Kaneda S, Ishizuka T, Sekiguchi A, Morimoto K, Kasukawa H. Efficacy of liposome-encapsulated hemoglobin in a rat model of cerebral ischemia. Artif. Organs 2014;38:650-655

9. Kawaguchi AT, Fukumoto D, Haida M, Ogata Y, Yamano M, Tsukada H. Liposome-encapsulated hemoglobin reduces the size of cerebral infarction in the rat: Evaluation with photochemically induced thrombosis of the middle cerebral artery. Stroke 2007;38:1626-1632 
10. Kawaguchi AT, Haida M, Ohba H, Yamano M, Fukumoto D, Tsukada H. Liposome-encapsulated hemoglobin ameliorates ischemic stroke in nonhuman primates: Longitudinal observation. Artif. Organs 2013;37:904-912

11. Kawaguchi AT, Haida M, Yamano M, Fukumoto D, Ogata Y, Tsukada H. Liposome-encapsulated hemoglobin ameliorates ischemic stroke in nonhuman primates: An acute study. J. Pharmacol. Exp. Ther. 2010;332:429-436

12. Urakami T, Kawaguchi AT, Akai S, Hatanaka K, Koide H, Shimizu K, et al. In vivo distribution of liposome-encapsulated hemoglobin determined by positron emission tomography. Artif. Organs 2009;33:164-168

13. Matsuo Y, Onodera H, Shiga Y, Nakamura M, Ninomiya M, Kihara T, et al. Correlation between myeloperoxidase-quantified neutrophil accumulation and ischemic brain injury in the rat. Effects of neutrophil depletion. Stroke 1994;25:1469-1475

14. Shimbo D, Abumiya T, Shichinohe H, Nakayama N, Kazumata K, Houkin K. Post-ischemic intra-arterial infusion of liposome-encapsulated hemoglobin can reduce ischemia reperfusion injury. Brain Res. 2014;1554:59-66

15. Longa E, Weinstein P, Carlson S, Cummins R. Reversible middle cerebral artery occlusion without craniectomy in rats. Stroke 1989;20:84-91

16. Garcia JH. Neurological deficit and extent of neuronal necrosis attributable to middle cerebral artery occlusion in rats. Statistical validation. Stroke 1995;26:627-634

17. Qin Z, Karabiyikoglu M, Hua Y, Silbergleit R, He Y, Keep RF, et al. Hyperbaric oxygen-induced attenuation of hemorrhagic transformation after experimental focal transient cerebral ischemia. Stroke 2007;38:1362-1367

18. Unekawa M, Tomita M, Tomita Y, Toriumi H, Miyaki K, Suzuki N. Rbc velocities in single capillaries of mouse and rat brains are the same, despite 10-fold difference in body size. Brain Res. 2010;1320:69-73

19. Ito U, Hakamata Y, Watabe K, Oyanagi K. Mannitol infusion immediately after reperfusion suppresses the development of focal cortical infarction after temporary cerebral ischemia in gerbils. Neuropathology 2014;34:360-369

20. Garcia JH LK, Yoshida Y, Chen S, Lian J. Brain microvessels: Factors altering their patency after the occlusion of a middle cerebral artery (wistar rat). American Journal of pathology 1994;145:728-740

21. Karibe H, Zarow GJ, Graham SH, PR W. Mild intraischemic hypothermia reduces postischemic hyperperfusion, delayed postischemic hypoperfusion, blood-brain barrier disruption, brain edema, and neuronal damage volume after 
temporary focal cerebral ischemia in rats. J Cereb Blood Flow Metab. 1994;14:620-627

22. Olah L WS, Hoehn M. Secondary deterioration of apparent diffusion coefficient after 1-hour transient focal cerebral ischemia in rats. J Cereb Blood Flow Metab. 2000;Oct;20:1474-1482

23. Jin R, Yang G, Li G. Inflammatory mechanisms in ischemic stroke: Role of inflammatory cells. J Leukoc Biol 2010;87:779-789

24. Lakhan SE. Matrix metalloproteinases and blood-brain barrier disruption in acute ischemic stroke. Frontiers in neurology 2013;4:32

25. Yamasaki Y, Matsuo Y, Matsuura N, Onodera H, Itoyama Y, Kogure K. Transient increase of cytokine-induced neutrophil chemoattractant, a member of the interleukin-8 family, in ischemic brain areas after focal ischemia in rats. Stroke 1995;26:318-322

26. Lerouet D, Beray-Berthat V, Palmier B, Plotkine M, Margaill I. Changes in oxidative stress, inos activity and neutrophil infiltration in severe transient focal cerebral ischemia in rats. Brain Res. 2002;958:166-175 


\section{Figure Legends}

Figure 1

Effects of the intra-arterial infusion of liposome-encapsulated hemoglobin (LEH) on neurological function and infarct/edema volumes. Neurological function evaluated using an 18-point scale $(n=8)$ at the onset of middle cerebral artery occlusion and after 24 hours of reperfusion (A). ${ }^{* *} \mathrm{P}<.01$.

(B) Evaluation of infarct and edema volumes with 2,3,5-triphenyltetrazolium chloride staining after 24 hours of reperfusion $(n=8) . * * \mathrm{P}<.01$.

Figure 2

Effects of the intra-arterial infusion of liposome-encapsulated hemoglobin (LEH) on neutrophil infiltration. (A) Representative images of myeloperoxidase (MPO) immunohistochemistry showing MPO-positive neutrophils in the perivascular space and parenchyma in the control, vehicle and LEH groups. (B) Representative image of MPO immunoblots and a bar graph showing the results of the quantitative analysis $(n=7) . * * \mathrm{P}<.01$ 


\section{Figure 3}

Effects of the intra-arterial infusion of liposome-encapsulated hemoglobin (LEH) on matrix metalloproteinase (MMP)-9 expression and activity. (A) Representative image of MMP-9 immunoblots and a graph showing the results of the quantitative analysis $(n=7)$. (B) Representative image of MMP-9 zymography and a graph showing the results of the quantitative analysis (n=7). $* \mathrm{P}<.05, * * \mathrm{P}<.01$

Figure 4

Effects of the intra-arterial infusion of liposome-encapsulated hemoglobin (LEH) on endothelial dysfunction represented by intercellular molecule-1 (ICAM-1) expression and Evans blue extravasation. (A) Representative image of ICAM-1 immunoblots and a graph showing the results of the quantitative analysis $(n=7)$. (B) Representative image of Evans blue extravasation observed in a brain hemisphere and a graph showing the results of the quantitative analysis $(n=6) . * \mathrm{P}<.05$, **P $<.01$ 


\section{Figure 5}

Perfusion of liposome-encapsulated hemoglobin (LEH) and autologous erythrocytes in microvessels in the ischemia reperfusion (I/R) region. (A) Representative images of immunohistochemistry with an anti-rat hemoglobin antibody in the control and vehicle groups and an anti-human hemoglobin antibody in the LEH group ( $(n=4)$. (B) A bar graph showing the results of the quantitative analysis on the number of hemoglobin-positive microvessels. (C) A bar graph showing the results of the quantitative analysis on the number of hemoglobin-positive microvessels less than $5 \mu \mathrm{m}$ in diameter.

$* * \mathrm{P}<.01$

Figure 6

Representative images of microvascular morphological changes in control and liposome-encapsulated hemoglobin (LEH) groups evaluated by transmission electron microscopy. Whereas microvessels with a swollen cytoplasm and microvilli was compressed and narrowed by swollen astrocyte end-feet (A, asterisks) in the control group, microvessels had a normal appearance and contained LEH particles (B, white arrows) and erythrocytes in the LEH group.

Scale bar $=2 \mu \mathrm{m}$. 
FIG. 1

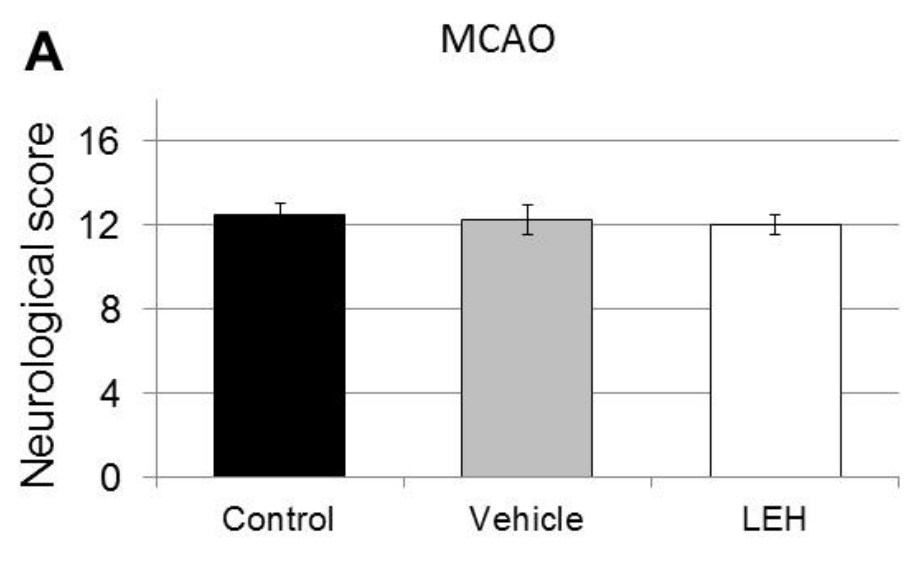

B

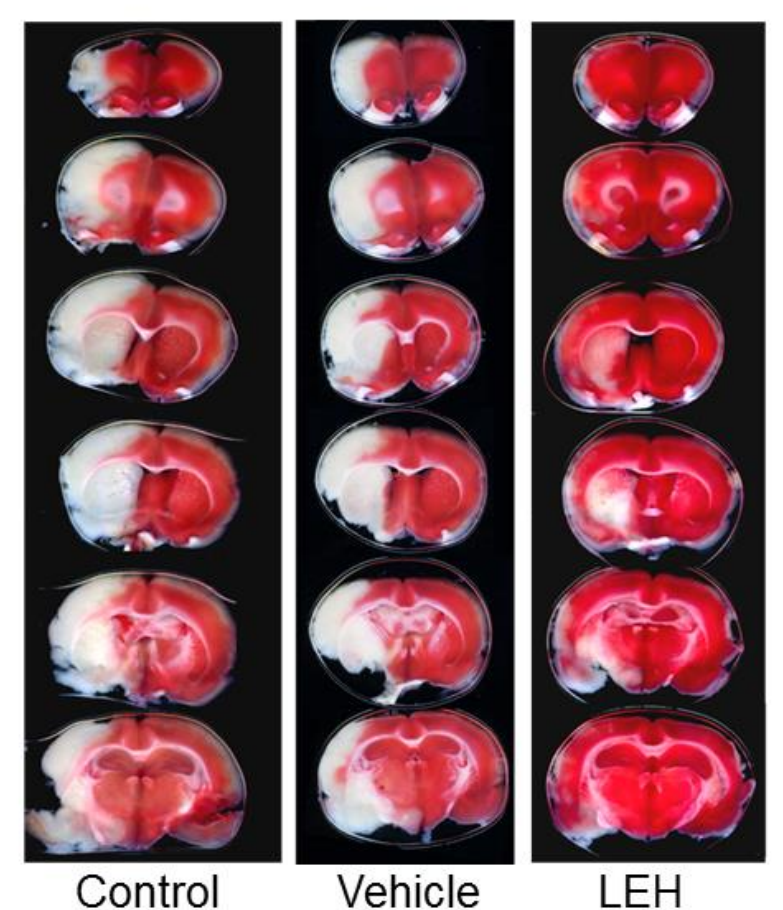

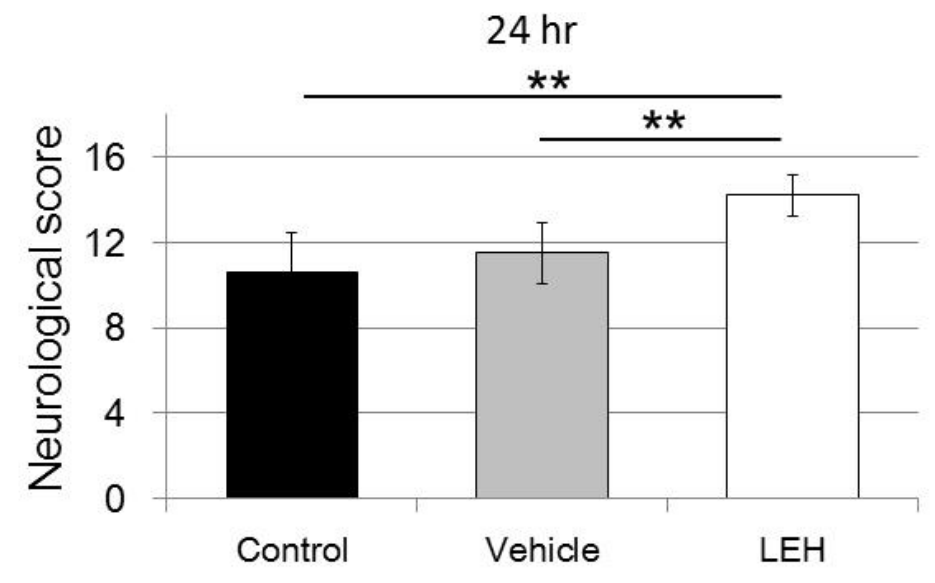
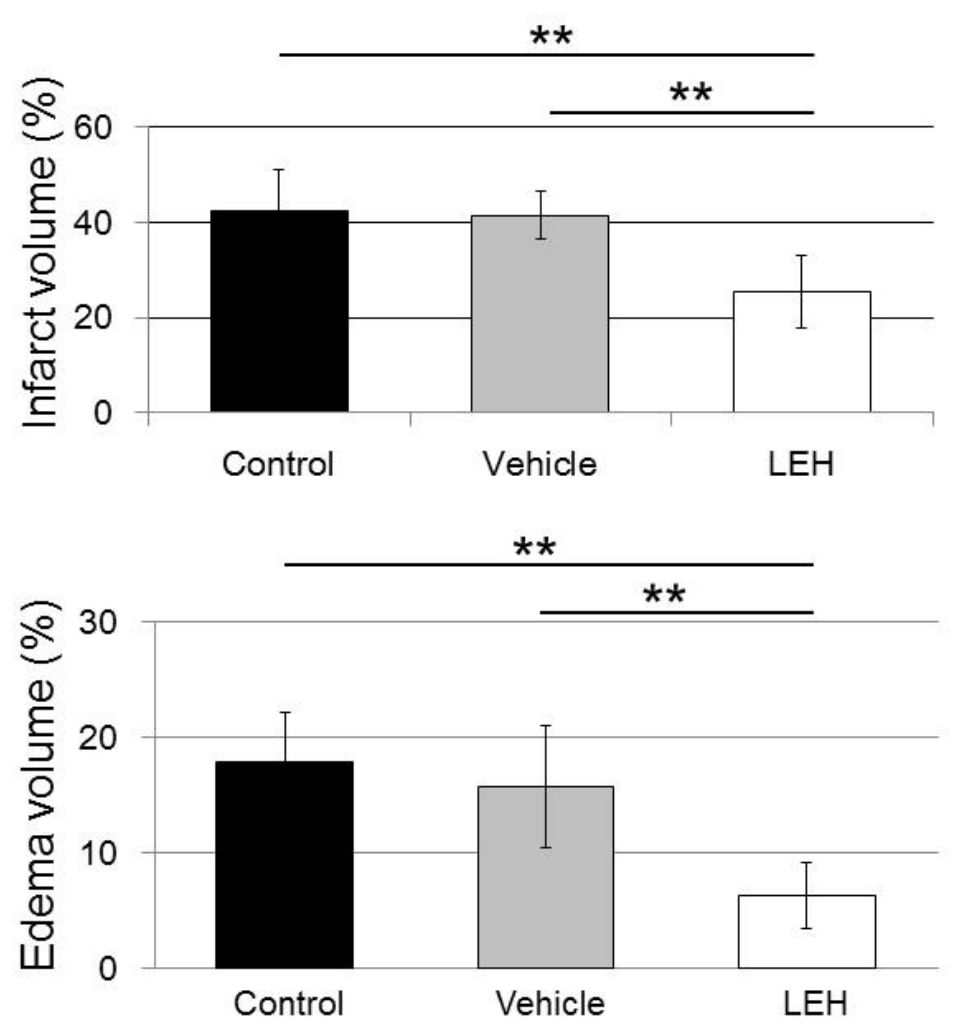
FIG. 2

A
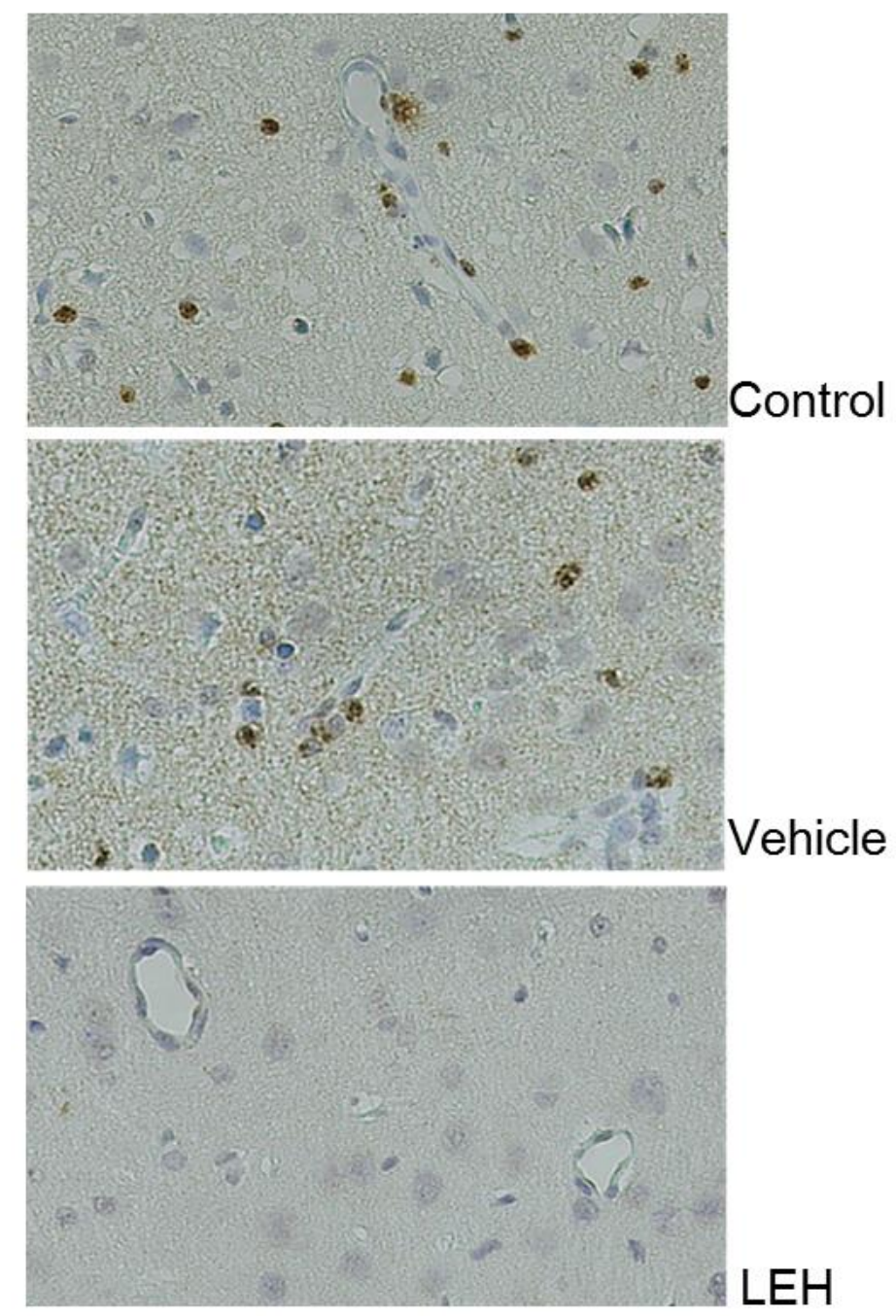

B

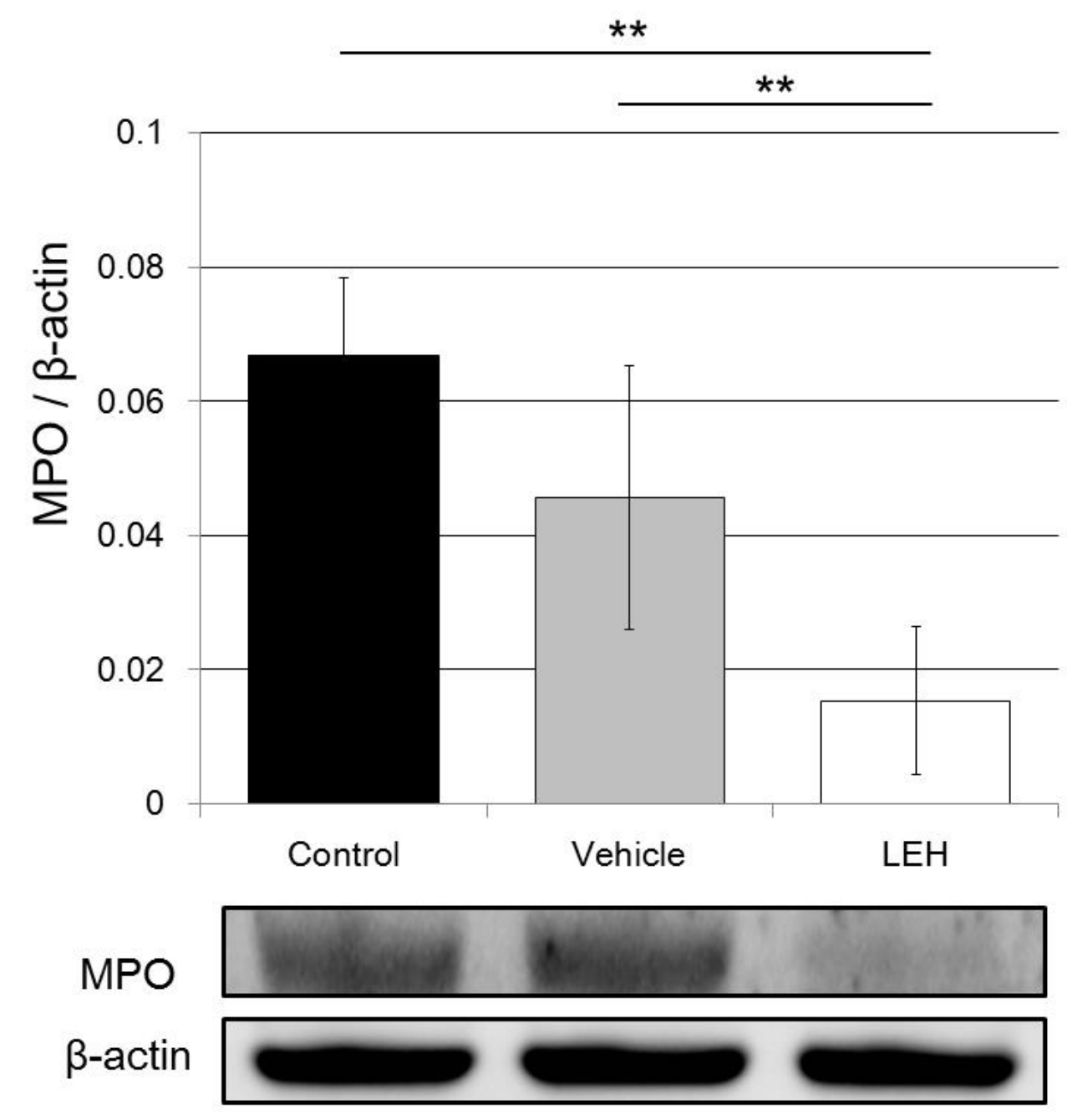


FIG. 3

A
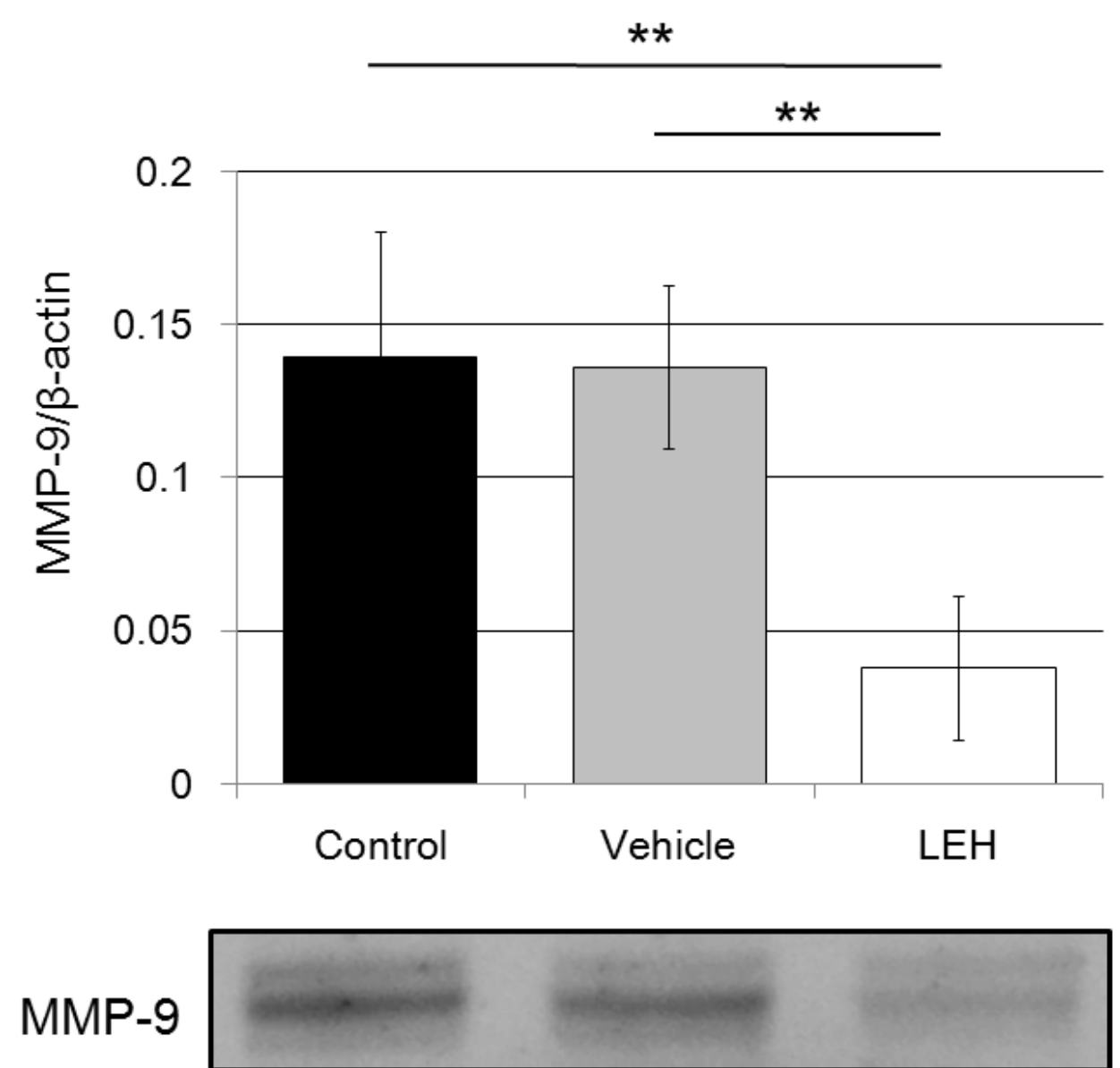

$\beta$-actin
B

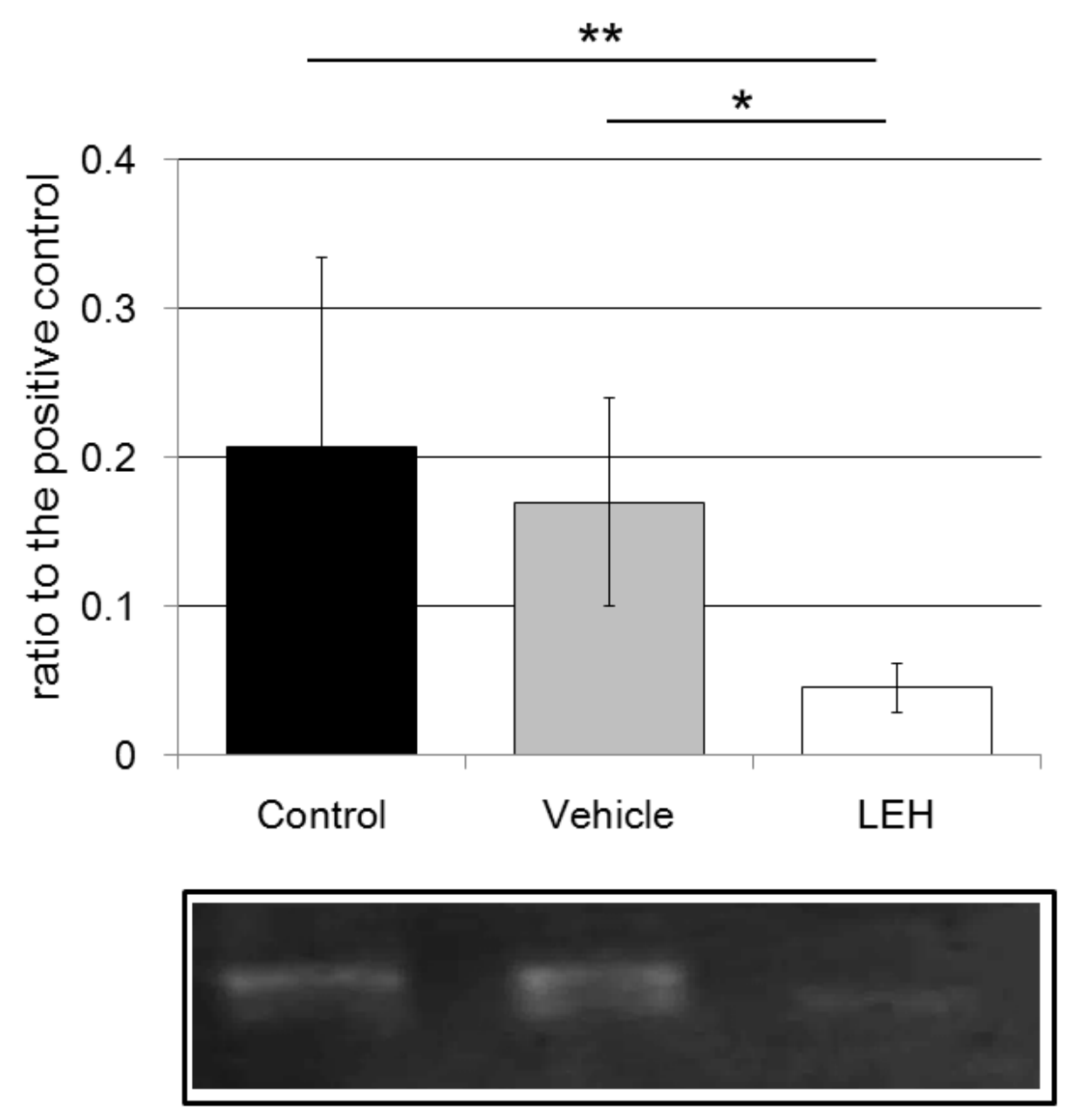


FIG. 4

A
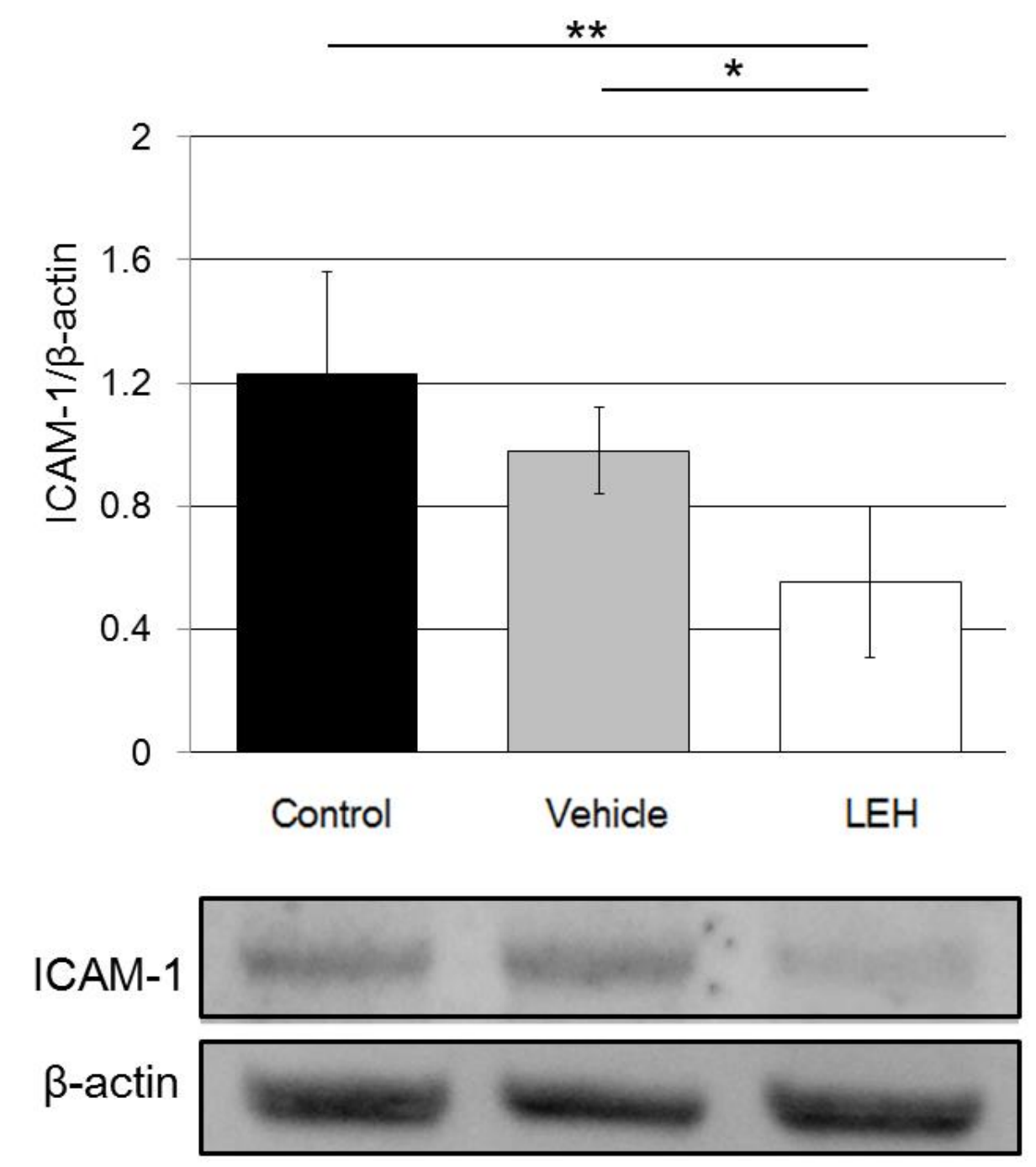

B
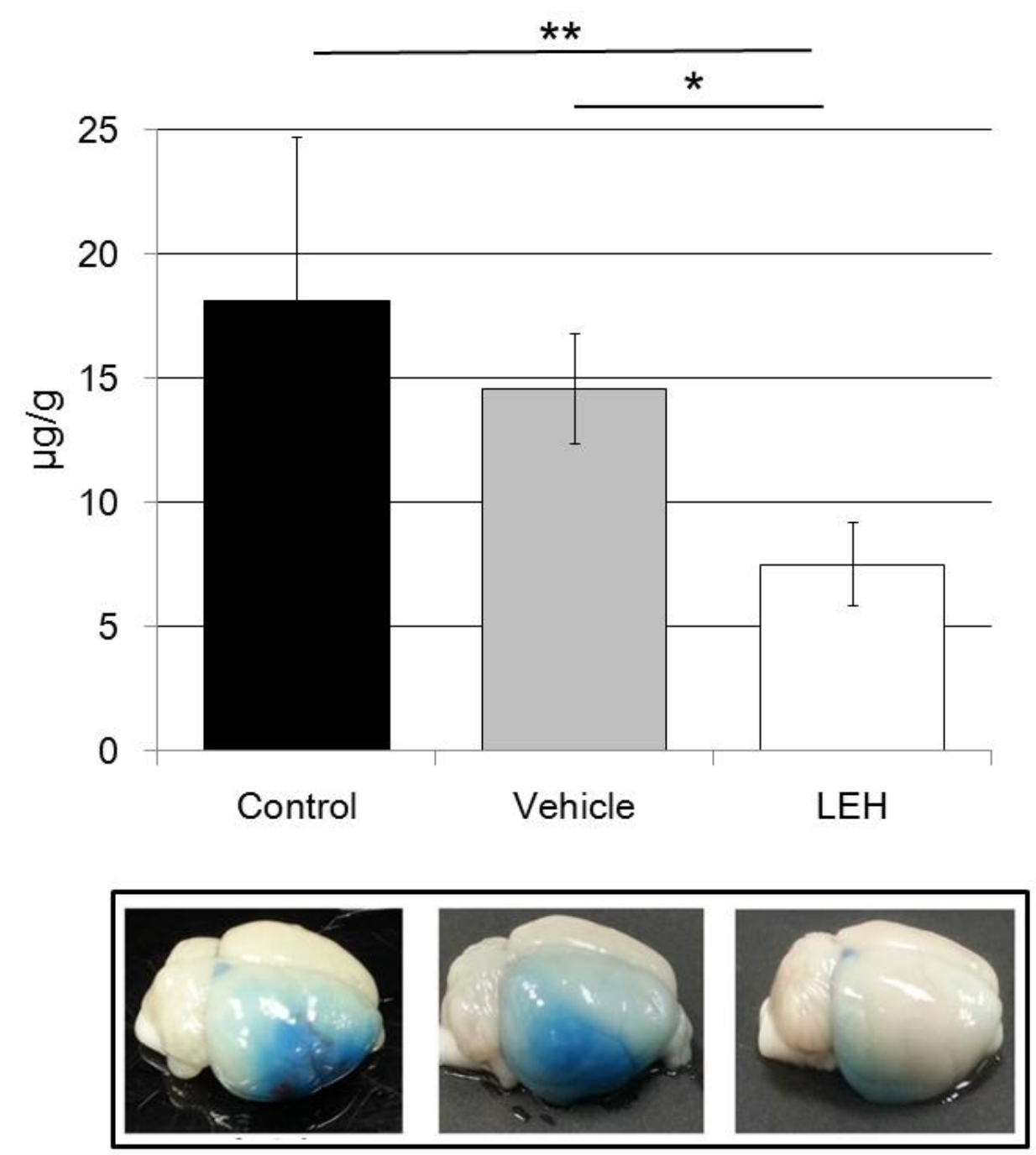
FIG. 5

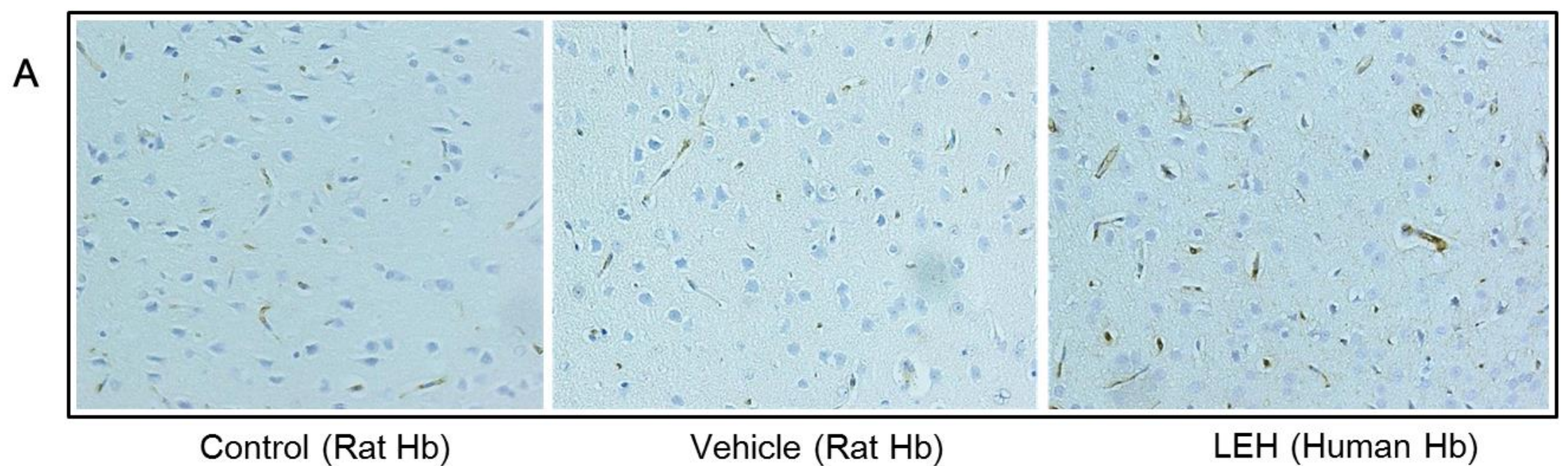

B

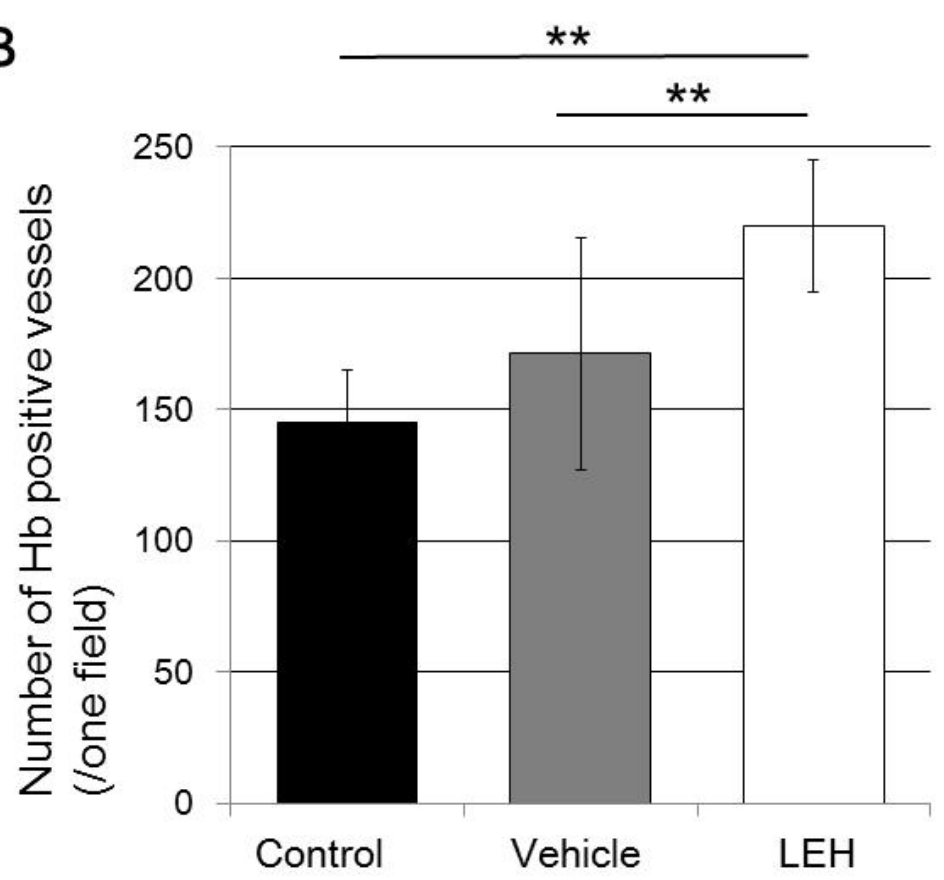

C

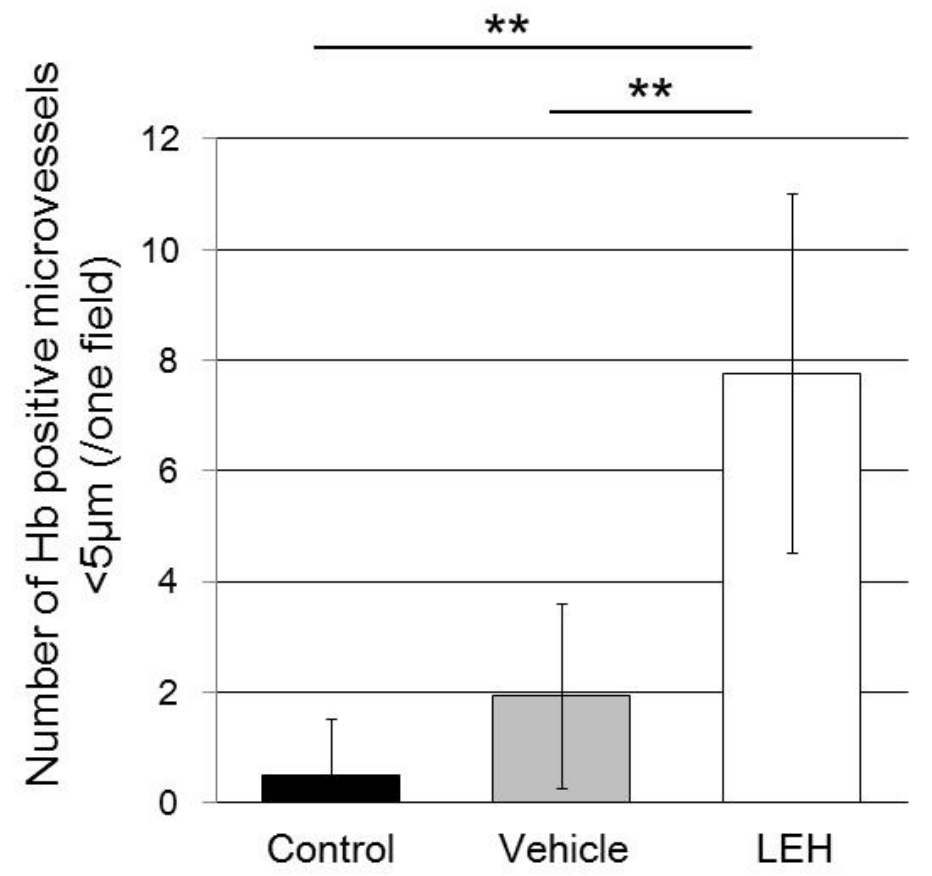


FIG. 6
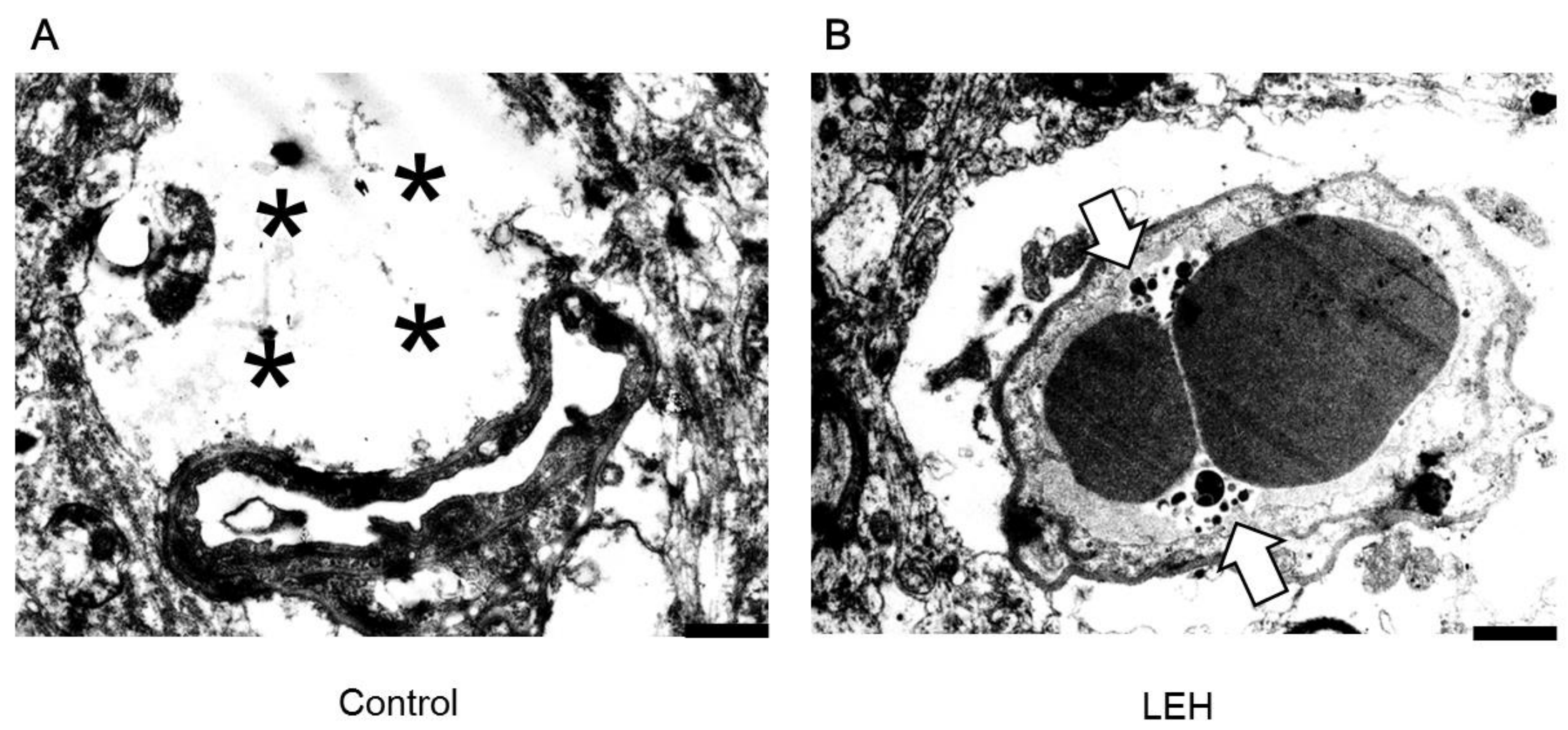
Table 1

\begin{tabular}{|c|c|c|c|c|c|}
\hline & & Control & Vehicle & LEH & \\
\hline \multirow[t]{6}{*}{ Pre MCAO } & Mean BP, mmHg & $100.5 \pm 3.9$ & $100.2 \pm 2.7$ & $102.1 \pm 3.7$ & n.s \\
\hline & Temperature, ${ }^{\circ} \mathrm{C}$ & $36.6 \pm 0.32$ & $36.4 \pm 0.24$ & $36.5 \pm 0.34$ & n.s \\
\hline & $\mathrm{pH}$ & $7.46 \pm 0.06$ & $7.44 \pm 0.038$ & $7.45 \pm 0.055$ & n.s \\
\hline & $\mathrm{pO} 2, \mathrm{mmHg}$ & $138.4 \pm 9.8$ & $140.7 \pm 10.8$ & $135 \pm 12$ & n.s \\
\hline & $\mathrm{pCO} 2, \mathrm{mmHg}$ & $30.4 \pm 4.4$ & $31.5 \pm 4.8$ & $31.4 \pm 7.1$ & n.s \\
\hline & $\mathrm{Ht}, \%$ & $39.8 \pm 1.8$ & $38.7 \pm 4.4$ & $39.7 \pm 2.1$ & n.s \\
\hline Reduction of $\mathrm{CBF}(\%)$ & & $24.2 \pm 5.2$ & $26.4 \pm 3.2$ & $25.2 \pm 3.8$ & n.s \\
\hline \multirow[t]{4}{*}{ After $24 \mathrm{~h}$ reperfusion } & $\mathrm{pH}$ & $7.52 \pm 0.067$ & $7.52 \pm 0.05$ & $7.54 \pm 0.04$ & n.s \\
\hline & $\mathrm{pO} 2, \mathrm{mmHg}$ & $136.5 \pm 15.9$ & $135.7 \pm 13.2$ & $138.3 \pm 16.4$ & n.s \\
\hline & $\mathrm{pCO} 2, \mathrm{mmHg}$ & $32 \pm 9.8$ & $32.5 \pm 4.3$ & $28.5 \pm 3.9$ & n.s \\
\hline & $\mathrm{Ht}, \%$ & $43.8 \pm 4.5$ & $43.1 \pm 3.4$ & $46 \pm 3.6$ & n.s \\
\hline
\end{tabular}

